Sinha B.K.

Ganesh Man Singh Memorial

Academy of ENT and Head \& Neck

Studies, Tribhuvan University Teaching

Hospital, Institute of Medicine,

Kathmandu, Nepal

Correspondence to:

Prof. B. K. Sinha

Professor and Head of Department

Department of ENT \& Head-Neck

Surgery, TUTH Kathmandu, Nepal.

E-mail:bimalsinha123@gmail.com

\section{SPLIT EAR LOBULE REPAIR}

Key words: Split ear Lobule, Z-Plasty
Split ear lobule repair is a procedure to repair a tear in the ear lobule and restore it to its original look, shape and function. Split ear lobule (Fig. 1) is a common condition in this part of the world where females wear a lot of heavy ear ornaments. Patients present either with a completely split lobule or with just an enlargement of the hole making it impossible for them to wear any ornaments.

There are several techniques with varying results, by which a split ear lobule can be repaired. The most commonly encountered post surgical repair complications are mild depression at the suture line and recurrence of the split. Some of the common techniques followed are described below.

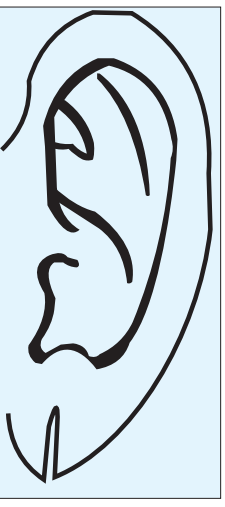

Fig. 1: Split ear lobule

Technique 1: After injecting the local anesthetic, incision is given with a no.11 or 15 blade along the entire length of the split surface of the lobule bisecting it and creating two flaps: medial and lateral on each of the split surfaces. The margins of the both sides of the split on both the medial and the lateral surfaces are sutured separately using a 5-0 non absorbable suture. Sutures are removed on $6^{\text {th }}$ or $7^{\text {th }}$ day. The drawbacks of this technique are that the suture line will deepen after some time and the chances of recurrence are very high.

Technique 2: After injecting the local anesthetic at the base of the lobule, the inner epitheliazed edge of skin is removed completely all around the split (fig. 2). Each fresh edge is then divided longitudinally for about $5 \mathrm{~mm}$. If it is not a complete split, the margin of the split surfaces are freshened by excising the skin all around the hole and/or the intact part of the lobule inferior to the hole is also split making the incomplete split a complete one depending upon the surgeon's choice. I prefer not to do it. After excising the skin of the margins, the medial and the lateral surfaces are undermined using a knife to about half a centimeter all around.

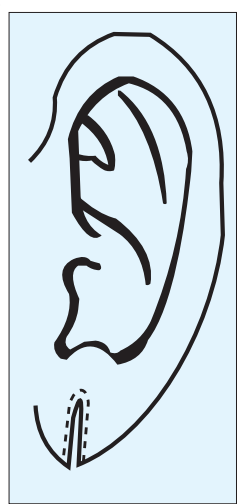

The medial and the lateral flaps are now sutured separately using a 5-0 non absorbable suture (Fig.3). The sutures are removed after 5-7days. The advantage of this technique is that there will be no depression along the suture line but the disadvantage is that the suture line is again not very strong and chances of recurrence of the split are very high.

Fig. 3: Refreshed margin sutured in a straight line

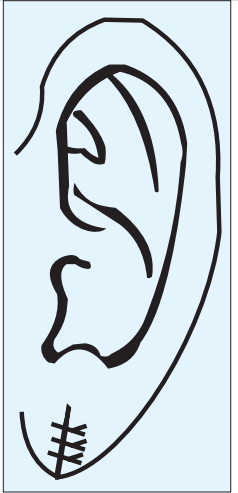

Technique 3 ( Z-plasty): Of all the described techniques this is the method that I prefer to use. In my opinion this is the best method of split ear lobule repair because it gives good strength to the scar, there will be no depression in the suture line and the chances of recurrence of split lobule are very less as the new hole does not lie on top of a straight suture line. It is a well known fact that flaps have less chance of scar notching.

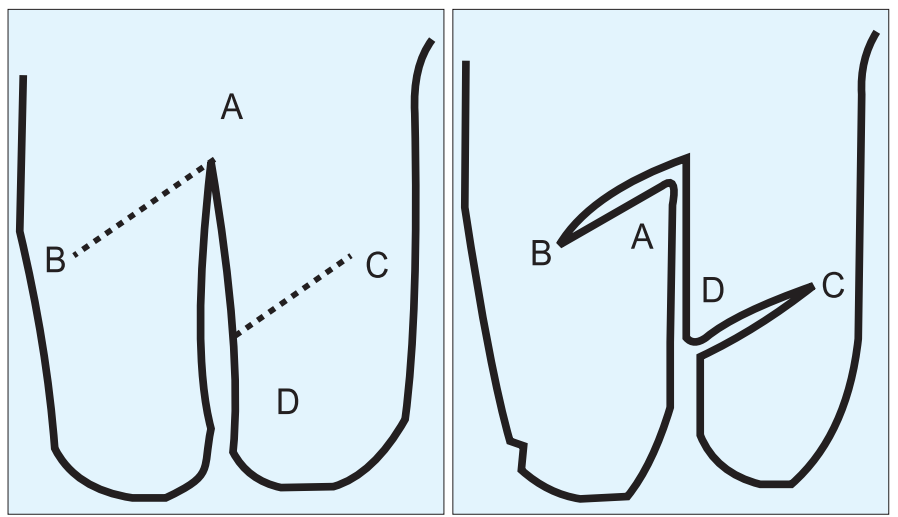

Fig.4 : Marking of the Z-Plasty incision line

Fig. 5: Incised lateral flap 


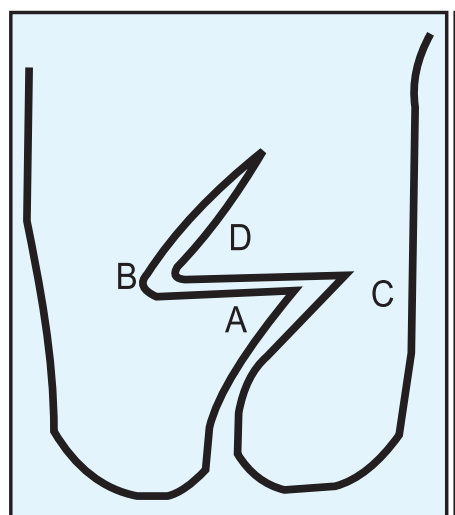

Fig.6: Joining of points $A$ to $\mathrm{C}$ and $\mathrm{D}$ to $\mathrm{B}$

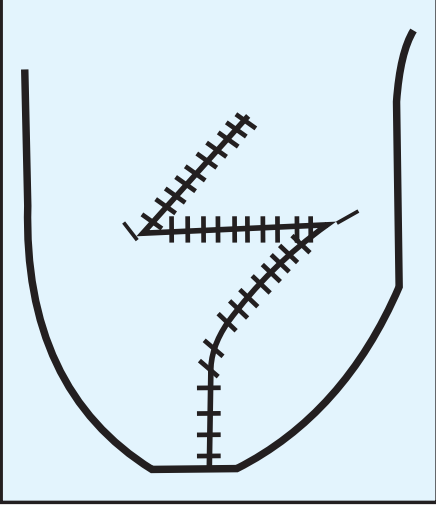

Fig. 7: Sutured lateral flap of Z-palsty
The initial steps of the surgery are the same as described in technique 2 , that is, freshening the margins by excising a rim of tissue from the free margins (fig.2) and then separating the medial and the lateral flaps longitudinally for about $5 \mathrm{~mm}$ using a knife. The uppermost point of the split $A$ is identified (Fig. 4) and so is another point $D$ at the free margin of the lateral flap about $0.5 \mathrm{~cm}$ from $A$. The length $A-D$ is measured and other points $B$ and $C$ are marked as shown in the diagram. The lengths of $A-D, A-B$ and $D-C$ should be equal. Then only the lateral flaps are cut joining the points $A-B$ and $C-D$ (Fig. 5). Suturing: If it is a complete split, the most important step is to approximate the tip of the lobule first very meticulously so that there is no stepping. The lateral flap is sutured next. Using a 5-0 prolene, the points $D$ to $B$ and $A$ to $C$ are joined (Fig.6). The rest of the lateral flap is sutured in a straight line (Fig.7). After completion of the suturing of the lateral flap, the flap on the medial surface is sutured in straight line. Piercing of the ear lobule is done at the time of the repair or later, at the uppermost point of the sutured part, using a straight cutting needle, which passes through the triangular flap of the Z plasty. However, wearing of ornaments should be avoided for about 6 weeks postoperatively. 